This item was submitted to Loughborough's Research Repository by the author.

Items in Figshare are protected by copyright, with all rights reserved, unless otherwise indicated.

\title{
Structural changes at grain boundaries in bcc iron induced by atomic
} collisions

PLEASE CITE THE PUBLISHED VERSION

LICENCE

CC BY-NC-ND 4.0

\section{REPOSITORY RECORD}

Perez, Francisco J. Perez, and Roger Smith. 2019. "Structural Changes at Grain Boundaries in Bcc Iron Induced by Atomic Collisions”. figshare. https://hdl.handle.net/2134/842. 


\title{
Structural changes at grain boundaries in bcc iron induced by atomic collisions
}

\author{
F. Javier Pérez-Pérez and Roger Smith \\ School of Mathematics \& Physics \\ Lougborough University \\ Leicestershire \\ LE11 3TU \\ UK.
}

November 22, 1999

\begin{abstract}
Symmetrical tilt and twist grain boundary structures have been simulated in bcc iron using a many-body potential of the Finnis-Sinclair form. Initial structures were relaxed to the local minimum energy configuration using molecular dynamics. The width and relative energies of the resulting grain boundaries have been calculated. Collision cascades have been initiated in the structure by imparting initial energy to a single Fe atom and the interaction of the cascade with the grain boundary has been studied again using molecular dynamics simulations. The cascades were chosen where the primary knock-on atom (PKA) had initial energy of 1 $\mathrm{keV}$ and the orientation and distance of the PKA were changed in order to generate some statistical information concerning the radiation damage near the interface. The results show an increased radiation damage in the grain boundary region compared to the bulk material. The interstitials that form in the boundary region seem to be stable and do not move away from the boundary during the recrystallisation phase of the collision cascade. Clusters of interstitials are easily produced at the boundary in either structure but the defects induced near the twist boundary are more extensive that those near the tilt boundary.
\end{abstract}

PACS numbers: 71.15.Pd, 61.80.-x, 61.72.Mn

KEYWORDS: molecular dynamics, bcc iron, grain boundaries, interfaces, radiation damage, collision cascades 


\section{Introduction}

The performance of steel components in nuclear reactors is significantly influenced by the presence of interfaces within the material such grain boundaries. Grain boundaries in polycrystalline materials, i.e. ferritic steels (bcc iron) used on nuclear reactors, affect a large variety of physical properties [1][2]. The process of neutron irradiation can degrade the physical properties of reactor core components [3], by introducing changes to the microstructure of the bulk and interfaces such as the introduction of point defects.

The analysis of these defect structures experimentally is quite difficult and atomistic simulations are an ideal alternative to provide an insight into the structure, changes and damage mechanisms near to grain boundaries under irradiation. Previous work on this topic includes a study of the atomic microstructure of interfaces in iron by atomistic simulations [4][5]. Also many features of mechanisms produced by radiation damage have been identified from simulations of collision cascades in initially perfect crystals of $\alpha$-iron via molecular dynamics $[6][7][8]$.

The natural step to improve the understanding of the role of grain boundaries in defect production is the study of collision cascades near these interfaces. Recently such simulations of radiation damage near interfaces in iron [9] and silver [10] have been reported.

This work describes the behaviour of radiation damage on two different grain boundaries in $\alpha$-iron. The two ideal grain boundaries chosen for this study are symmetrical tilt and symmetrical twist boundaries. There are two steps in modelling process. The first step previous to the calculation of radiation damage near these grain boundaries, is to model the static microstructure for a better understanding of the atomic structure at the interface of two matching crystals. The second step involves the simulation of collision cascades on bicrystals containing theses interfaces in order to generate enough statistics for comparison. 


\section{Model}

The different ways that two crystals can meet to produce a grain boundary are defined by five macroscopic parameters. Following the coincident site lattice (CSL) misorientation scheme the five macroscopic degrees of freedom are the rotation axis in the direction of the unit vector, $\hat{n}$, the misorientation angle, $\phi$, and the grain boundary plane normal, $\hat{n}_{1}$, in either of the two halves of the bi-crystal [11]. Following this parameterisation, a pure tilt boundary is defined as the boundary created with a rotation axis which is perpendicular to the grain boundary plane normal $\hat{n} \perp \hat{n}_{1}$. Also, a pure twist boundary is created when the rotations axis is parallel to the grain boundary plane normal $\hat{n} \| \hat{n}_{1}$. They are called symmetric grain boundaries if $\hat{n}_{1}$ and $\hat{n}_{2}$ have the same Miller index form. Following the CSL scheme we can create a bi-crystal with the boundary required as a starting point for a relaxation molecular dynamics procedure. In the case of a symmetrical tilt boundary we also add a rigid body translation parallel to the boundary to make a more stable initial configuration as is explained in [9]. The unrelaxed structure is assumed to be at $0 \mathrm{~K}$ but containing excess potential energy.

In order to represent the interaction between iron atoms, a many-body potential for $\alpha$-iron has been used. This potential has the Finnis-Sinclair form [12] with modifications on the ion-ion repulsive part with a view to giving a good description of the collision event at high energies [6].

The unrelaxed structures are allowed to evolve until the block reaches a minimum energy configuration at a given temperature using classical molecular dynamics (MD). The final temperature of the block calculated from the mean kinetic energy of the atoms was fixed to $10 \mathrm{~K}$. The computational blocks employ periodic boundary conditions in the planes parallel to the grain boundary, i.e. the $\mathrm{x}$ and $\mathrm{z}$ directions. Free boundary conditions are applied in the $y$ direction, perpendicular to the boundary plane. The computational technique was to run the MD simulation for 3 ps at what time the kinetic and potential energies are equipartitioned. Then a frictional term proportional to the velocity of the 
atoms is introduced. The simulations were then run for typically another $10 \mathrm{ps}$ until the temperature dropped to $10 \mathrm{~K}$ and the equilibrium configuration was reached.

Collision cascades are generated on the relaxed computational blocks obtained by the relaxation procedure. In order to generate a collision cascade an excess of kinetic energy is imparted to an atom chosen from the relaxed computational block. The evolution of this system is studied using molecular dynamics simulations. The simulation is allowed to evolve until reaching a stable state with no significant evolution in the number of point defects created by the collision cascade, i.e. typically 5 ps with $1 \mathrm{keV}$ imparted to the PKA. Then, the kinetic energy of the system is reduced by introducing a damping proportional to the velocity of the atoms until the temperature of the system drops to $10 \mathrm{~K}$, typically 1-2 ps more. The definition of interstital and vacancies are as describe in reference [9]. It should be noticed that we define interstitials and vacancies on the interface in the same way as in the bulk, considering the atomic sites from the relaxed structure of the boundary.

\section{Results}

\subsection{Grain Boundary Microstructure}

Two rectangular computational cells have been made to study the relaxed structure and radiation effects, each of them simulating two different symmetrical boundaries, tilt and twist. The interface between both crystals making the bicrystal is placed on the middle of the computational cell, with the boundary plane parallel to the $\mathrm{x}-\mathrm{z}$ plane. In Table 1 boundary plane normals for both bicrystals, $\hat{n}_{1}$ and $\hat{n}_{2}$, the misorientation angle, $\phi$, the rotation axis, the coincident site lattice parameter, $\Sigma$, are presented. In addition, the size of the computational cell and the number of atoms contained in each model are also given.

Figure 1 shows the final relaxed structure of the [001] $28.073^{\circ}(530)$ symmetrical tilt boundary. In Figure 1 (a), a projection of the atoms close to the grain 
boundary in a plane perpendicular to the boundary, i.e. plane $\mathrm{x}-\mathrm{y}$, is shown. Figure 1 (b) shows atomic sites close to the grain boundary projected onto a plane parallel to the boundary. From these figures, we clearly can see the periodicity of the structure in the direction parallel to the boundary labelled here as the $\mathrm{x}$ and $\mathrm{z}$ directions. Also, the structure shows a clear symmetry on the $\mathrm{y}$ direction, perpendicular to the boundary plane, across the interface. Figures 2 (a) and (b), show the projection of the atomic sites close to the boundary on planes perpendicular and parallel to the boundary itself respectively, of the [001] $28.073^{\circ}$ (001) symmetrical twist boundary. Also, the symmetrical twist shows a periodicity structure similar to the Moiré patterns [13] on planes parallel to the boundary, i.e. directions $\mathrm{x}$ and $\mathrm{z}$.

\subsection{Grain Boundary Energy and Width}

As has been shown in the previous section, the regular crystal structure of both crystals is broken by a disordered region of only a few atomic layers. Because of this region the physical properties, i.e. thermal expansion, electrical resistivity, elastic response, in the vicinity of the interface can differ substantially from those of the rest of the bulk and can be highly anisotropic. These differences can change over a few atomic layers in different ways, dependent on the physical property, therefore to define the width of a grain boundary is not an easy task.

A definition of the width of a grain boundary can be made, based on our atomistic model using the potential energy of the atoms close to the boundary. Atoms close to the boundary will have a potential energy which differ clearly from those atoms from the bulk. The cohesive energy of an iron atom in a bcc crystal is equal to $-4.28 \mathrm{eV}$. The calculations predict a potential energy per atom equal to this cohesive energy in the undisturbed bulk. We define the width of the grain boundary as the region near the diving interface which includes atoms with a potential energy not in the interval $-4.28 \pm 0.01 \mathrm{eV}$.

In Figure 3 the average potential energy of atoms belonging to thin slabs of the computational cell as a function of the perpendicular distance from the grain 
boundary is shown for both symmetrical tilt and twist boundaries. Following our definition the width of the [001] $28.073^{\circ}$ (530) symmetrical tilt is approximately of $17 \AA$, whereas the width of the [001] $28.073^{\circ}$ (001) symmetrical twist is approximately of $8 \AA$. In Figure 3, the large values of the potential energy far away from the boundary correspond to the edges of the computational cell, because of the free boundary conditions considered on the direction perpendicular to the interface.

Once the width of the grain boundary is defined, the grain boundary energy can be calculated in the following way. We define the grain boundary energy per unit area, $\gamma$, as the difference between the potential (configurational) energy of $n$ atoms belonging to a grain boundary, i.e. atoms in the width, $E_{p}^{g b}$, and the potential energy of a computational cell with the same number of atoms in a perfect crystal, $E_{p}^{0}$, divided by the cross-sectional area of the grain boundary plane.

$$
\gamma=\frac{E_{p}^{g b}-E_{p}^{0}}{\text { Area }}
$$

The zero energy is attributed to the perfect single crystal configuration which is the reference state, therefore $E_{p}^{0}=-4.28 n$. With this definition we obtain values of $0.080 \mathrm{eV} / \AA^{2}$ for the grain boundary energy of the [001] $28.073^{\circ}$ (530) symmetrical tilt boundary and $0.121 \mathrm{eV} / \AA^{2}$ for the grain boundary energy of the [001] $28.073^{\circ}(001)$ symmetrical twist boundary.

These values are $20-30 \%$ bigger than the values for the same grain boundary energies calculated by D. Wolf using a Johnson's pair potential [5][11]. This fact is also noticed by R. Watanabe et al.[14], after structural calculations of different tilt boundaries in bcc iron.

\subsection{Collision Cascades}

In this section the effect of collision cascades near the two grain boundaries considered are presented. The starting configurations chosen for the simulation 
of collision cascades are the relaxed structures described in the previous sections. The collision cascade simulation starts when an excess of kinetic energy is imparted to an atom, the PKA (Primary Knock-on Atom). In order to generate different statistics, various velocity orientations for the PKA are chosen and different PKA's are chosen. Table 2 and Table 3 show the collision cascades considered for the tilt boundary and the twist boundary respectively. In these tables the velocity direction, and the distance perpendicular to the grain boundary of the PKA are presented with a minimum of 3 different choices of PKA for each velocity considered. An overall total of 30 collision cascades have been simulated for the tilt boundary and 15 for the twist boundary.

The behaviour of the collision cascade is similar to a typical collision cascade in a perfect crystal. The biggest change of energies is produced during the first half picosecond, where the kinetic energy excess of the PKA is transmitted to the system. After this time the system does not suffer large energy fluctuations. After 5 picoseconds, we introduce a damping to reduce the kinetic energy of the system until the system reaches $10 \mathrm{~K}$ of temperature.

In Figure 4 the number of point defects as a function of the simulation time is presented for both, symmetrical tilt and symmetrical twist boundaries. It is clearly shown that the maximum number of defects is produced during the firsts half picosecond for both systems. After the first picosecond most of the defects relaxed and we consider that we have stable defects after about 6 picoseconds of the simulation time. From Figure 4, we conclude that the number of final point defects in this symmetrical tilt boundary produced by a $1 \mathrm{keV}$ collision cascades is approximately four times the number of point defects of the same kind of collision cascades in a symmetrical twist boundary. Figures 5 and 6 show different stages of the simulation cascade for the symmetrical tilt and twist respectively for an initial PKA direction normal to the boundary. The initial distance of the PKA from the boundary is approximately $26 \AA$ in both cases. The cascades show similar features to those initiated in a perfect crystal with replacement collision cascades. The three dimensional nature of the pictures is a little difficult to interpret but it appears that most of the replacement collision cascades 
do not permeate through the boundary. Generally the boundary region acts as a region where defects build-up. These occur either as a result of rearrangement of existing interface atoms or as a build-up of interstitials arising from outside the boundary region. Approximately $90 \%$ of defects produced for the twist boundary model lie inside the grain boundary region. The corresponding figure for the tilt boundary model is $70 \%$.

The results show that there is a relationship between number of final defects and the PKA direction and distance from the boundary. It appears that for the twist boundary, the number of defects is maximum when the initial PKA is located between $15 \AA$ and $20 \AA$ from the interface. The distance is somewhat higher for the tilt boundary. As the PKA distance is increased the number of induced defects reduces to that for the perfect initial crystal.

Defect clustering occurs in the grain boundary region. In the tilt model, dumbells are the most frequent defects, and cluster of up to 6 interstitials are found. In the twist model clusters of up to 9 interstitials are found.

After relaxation, the energy of the tilt grain boundary is increased by about $5 \%$ whilst in the case of the twist model the energy is decreased by about $2.5 \%$. This suggests that the atomic configuration of the twist boundary might not be optimal as we would expect an increase in energy after radiation. The twist boundary has a higher initial energy than the tilt boundary and the density of atoms is less there. There could be two reasons why the twist boundary has this higher energy. The first reason could be that there are insufficient atoms at the interface to form the best minimum energy structure. This aspect could be studied further by adding atoms to the interface region and relaxing until a lower energy is reached. The second aspect could be that the method chosen for relaxing the interface for the twist boundaries does not produce the optimal configuration. This could be checked by allowing more random positional changes to atoms in the grain boundary region and relaxing theses structures. However neither procedure was adopted in this preliminary study. 


\section{Acknowledgement}

This research was supported by a Training and Mobility of Researchers Grant of the EEC, as a part of the EURATOM research program in the Nuclear Fission Safety-Reactor Safety area. The authors would also like to thank Rachel Thomson for useful discussion and encouragement.

\section{References}

[1] P. Lejček and S. Hofmann, Critical Reviews in Solid State and Materials Sciences 20 (1995) 1.

[2] A. P. Sutton and R. W. Ballufi, Interfaces in Crystalline Materials (Oxford University Press, 1996).

[3] W. J. Phythian and C. A. English, Journal of Nuclear Materials 205 (1993) 162.

[4] M. Hashimoto, Y. Ishida, R. Yamamoto and M. Doyama, Acta Metallurgica 32 (1984) 11.

[5] D. Wolf, Philosophical Magazine A 62 (1990) 4447.

[6] A. F. Calder and D. J. Bacon, Journal of Nuclear Materials 207 (1993) 25.

[7] R. E. Stoller, G. R. Odette and B. D. Wirth, Journal of Nuclear Materials 251 (1997) 49.

[8] R. Vascon and N. V. Doan, Radiation Effects and Defects in Solids 141 (1997) 375.

[9] F. J. Pérez-Pérez and R. Smith, Nuclear Instruments and Methods B 153 (1999) 136.

[10] K. Sugio, Y. Shimomura and T. D. de la Rubia, Journal of the Physical Society of Japan 67 (1998) 3882 . 
[11] D. Wolf and K. L. Merkle, Materials Interfaces. Atomic-Level Structure and Properties (Chapman \& Hall, London, 1992) pp. 87-150, pp. 87-150.

[12] M. W. Finnis and J. E. Sinclair, Philosophical Magazine A 50 (1984) 145.

[13] W. Bollmann, General Geometrical Theory of Crystalline Interfaces (Springer Verlag, Berlin, 1970).

[14] R. Watanabe, A. Nogami and T. Matsumiya, Materials Science Forum 204-206 (1996) Pt1-2 227. 


\section{Tables}

\begin{tabular}{|l|l|l|l|l|l|}
\hline Plane normals & $\phi$ (degrees) & Rotation axis & $\Sigma$ & Block size $(\AA)$ & Atoms \\
\hline$(530)(350)$ & 28.073 & {$[001]$} & 5 & $104 \times 104 \times 42$ & 36,900 \\
\hline$(001)(001)$ & 28.073 & {$[001]$} & 5 & $84 \times 71 \times 84$ & 42,500 \\
\hline
\end{tabular}

Table 1: Details of the two symmetrical boundary models considered

\begin{tabular}{|c|c|}
\hline $\begin{array}{l}\text { PKA } \\
\text { Directions }\end{array}$ & $\begin{array}{l}\text { PKA distance from } \\
\text { the interface }(\AA)\end{array}$ \\
\hline$[530]$ & $\begin{array}{cccccc}9.5 & 17.9 & 26.3 & 31.7 & 38.0 & 43.0\end{array}$ \\
\hline$[350]$ & $\begin{array}{lllll}7.6 & 9.5 & 13.5 & 17.9\end{array}$ \\
\hline [001] & $\begin{array}{llll}6.1 & 9.5 & 13.0 & 18.4 \\
\end{array}$ \\
\hline [100] & $\begin{array}{llll}7.6 & 10.5 & 13.5 & 20.8 \\
\end{array}$ \\
\hline [210] & $\begin{array}{llll}8.0 & 9.5 & 13.5 & 14.9\end{array}$ \\
\hline [110] & $\begin{array}{llll}6.5 & 7.6 & 9.5 & 9.5\end{array}$ \\
\hline [111] & \begin{tabular}{llll|}
14.5 & 17.4 & 19.9 & 22.8 \\
\end{tabular} \\
\hline
\end{tabular}

Table 2: Collision cascades considered near the symmetrical tilt boundary

\begin{tabular}{|l|ccc|}
\hline $\begin{array}{l}\text { PKA } \\
\text { Directions }\end{array}$ & \multicolumn{3}{|c|}{$\begin{array}{l}\text { PKA distance from } \\
\text { the interface }(\AA)\end{array}$} \\
\hline$[010]$ & 14.2 & 8.5 & 25.7 \\
\hline$[001]$ & 2.0 & 8.4 & 17.1 \\
\hline$[210]$ & 5.6 & 11.3 & 15.7 \\
\hline$[110]$ & 8.4 & 15.6 & 22.8 \\
\hline$[111]$ & 7.0 & 11.3 & 17.1 \\
\hline
\end{tabular}

Table 3: Collision cascades considered near the symmetrical twist boundary 


\section{$5 \quad$ Figure Captions}

Figure 1. View of the relaxed symmetrical tilt boundary region, [001] 28.073 (530). a) (001) projection, i.e. $x-y$ plane of the computational block. b) (35̄0) projection, i.e. z-y plane of the computational block.

Figure 2. View of the relaxed symmetrical twist boundary region, [001] 28.073 (001). a) (350) projection, i.e. y-z plane of the computational block. b) (001) projection, i.e. $\mathrm{x}-\mathrm{z}$ plane of the computational block.

Figure 3. Average potential energy of atoms included in thin slabs parallel to the grain boundary plane as a function of the coordinate perpendicular to the interface. Open circles are the values for the symmetrical tilt boundary and solid squares for the symmetrical twist boundary.

Figure 4. Number of point defects, vacancies and interstitials, generated for $1 \mathrm{keV}$ collision cascades as a function of the simulation time. a) Symmetrical tilt boundary. b) Symmetrical twist boundary. The two curves in (a) and (b) represent the upper and lower limits for the number defects from the simulations carried out.

Figure 5. Various stages in the development of a typical $1 \mathrm{keV}$ collision cascade near the [001] $28.073^{\circ}$ (530) symmetrical tilt boundary at normal incidence to the boundary. The dark circles represent vacancies and the lighter circles interstitials. a) $0.05 \mathrm{ps}$; b) $0.225 \mathrm{ps}$; c) $0.5 \mathrm{ps}$; d) $6 \mathrm{ps}$.

Figure 6. Various stages in the development of a typical $1 \mathrm{keV}$ collision cascade near the [001] $28.073^{\circ}$ (001) symmetrical twist boundary at normal incidence to the boundary. The dark circles represent vacancies and the lighter circles interstitials. a) $0.05 \mathrm{ps}$; b) $0.225 \mathrm{ps}$; ) $0.5 \mathrm{ps}$; d) $6 \mathrm{ps}$. 

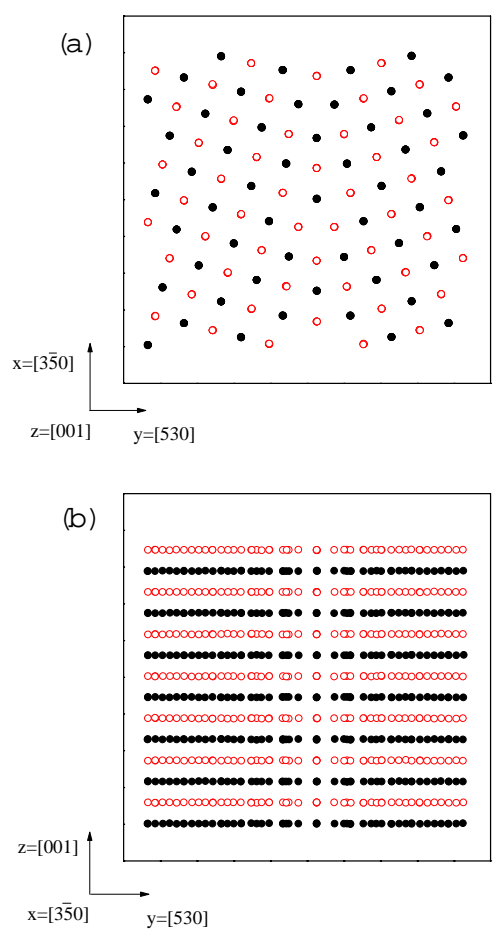

Figure 1: View of the relaxed symmetrical tilt boundary region, [001] 28.073 ${ }^{\circ}$ (530). a) (001) projection, i.e. x-y plane of the computational block. b) (35̄0) projection, i.e. z-y plane of the computational block. 

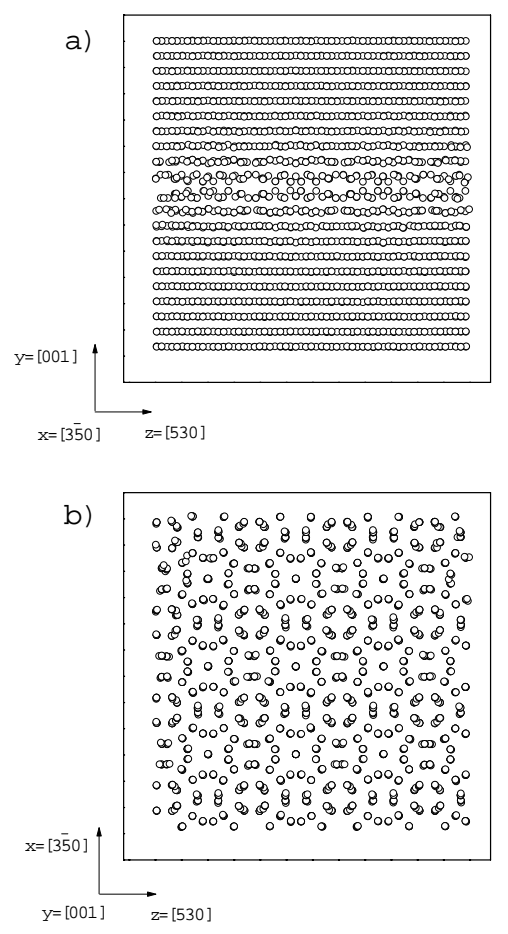

Figure 2: View of the relaxed symmetrical twist boundary region, [001] 28.073 (001). a) (350) projection, i.e. y-z plane of the computational block. b) (001) projection, i.e. $\mathrm{x}-\mathrm{z}$ plane of the computational block. 


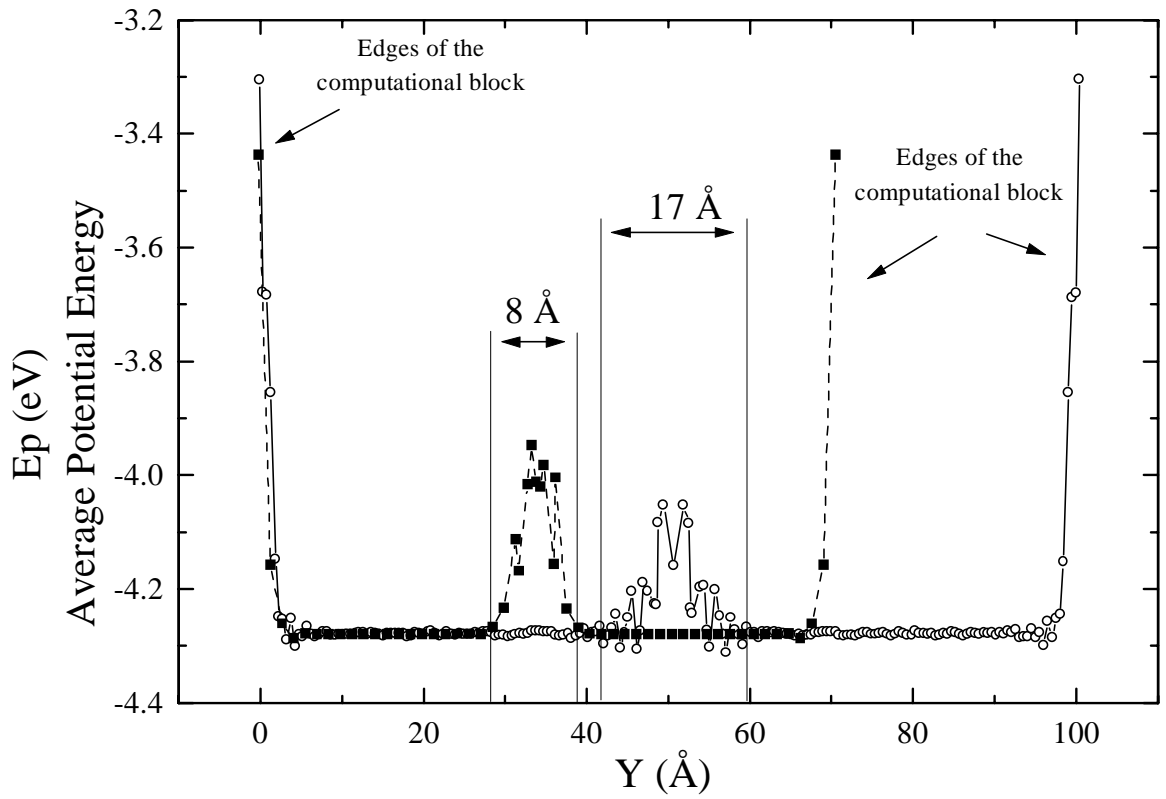

Perpendicular to the Boundary Plane

Figure 3: Average potential energy of atoms included in thin slabs parallel to the grain boundary plane as a function of the coordinate perpendicular to the interface. Open circles are the values for the symmetrical tilt boundary and solid squares for the symmetrical twist boundary. 

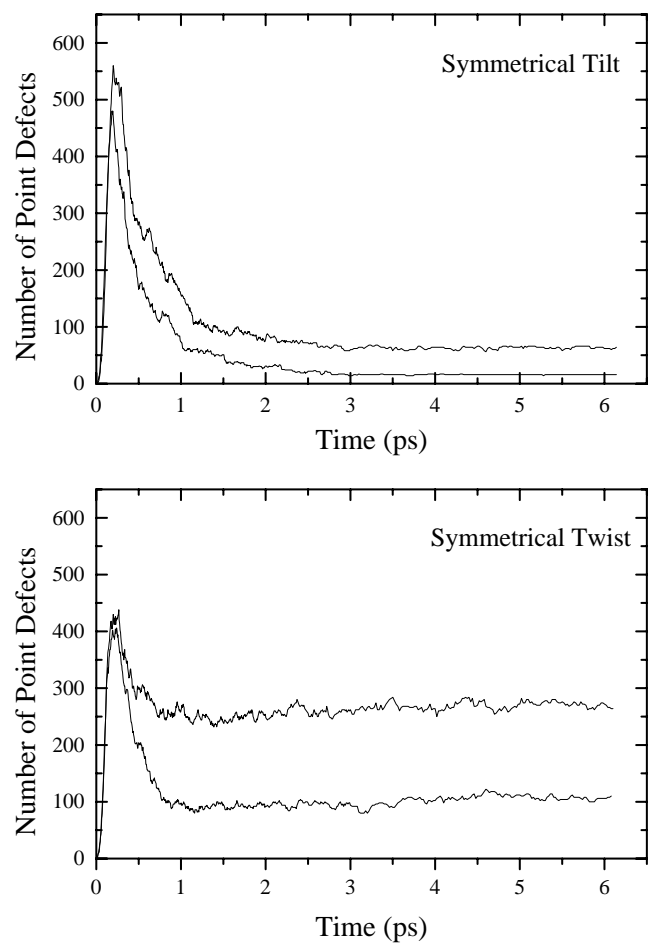

Figure 4: Number of point defects, vacancies and interstitials, generated for $1 \mathrm{keV}$ collision cascades as a function of the simulation time. a) Symmetrical tilt boundary. b) Symmetrical twist boundary. The two curves in (a) and (b) represent the upper and lower limits for the number defects from the simulations carried out 


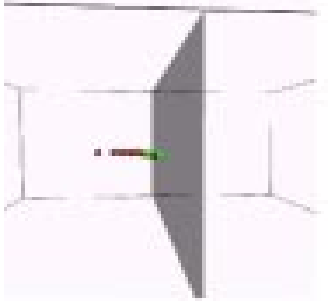

a)

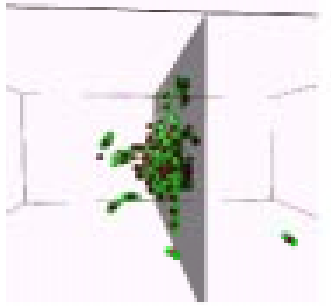

c)

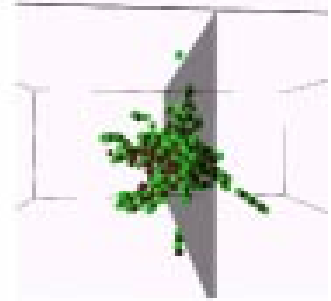

b)

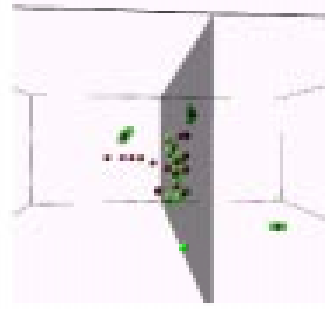

d)

Figure 5: Various stages in the development of a typical $1 \mathrm{keV}$ collision cascade near the [001] $28.073^{\circ}$ (530) symmetrical tilt boundary at normal incidence to the boundary. The dark circles represent vacancies and the lighter circles interstitials. a) $0.05 \mathrm{ps}$; b) $0.225 \mathrm{ps}$; c) $0.5 \mathrm{ps}$; d) $6 \mathrm{ps}$. 


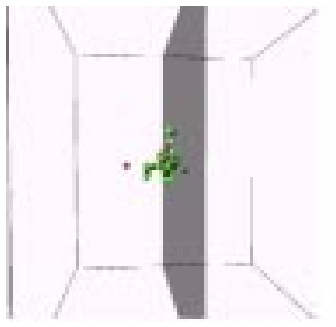

a)

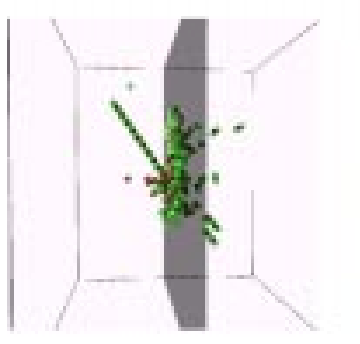

c)

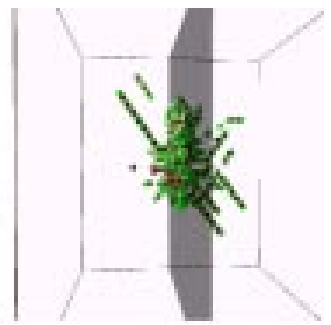

b)

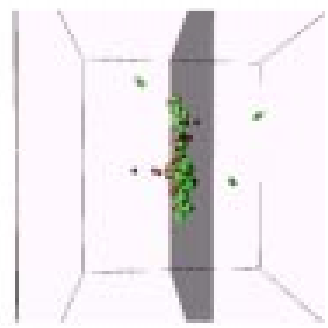

d)

Figure 6: Various stages in the development of a typical $1 \mathrm{keV}$ collision cascade near the [001] $28.073^{\circ}$ (001) symmetrical twist boundary at normal incidence to the boundary. The dark circles represent vacancies and the lighter circles interstitials. a) $0.05 \mathrm{ps}$; b) $0.225 \mathrm{ps}$; c) $0.5 \mathrm{ps}$; d) $6 \mathrm{ps}$. 